\title{
Homological actions on sutured Floer homology
}

\author{
YI Ni
}

\begin{abstract}
We define the action of the homology group $H_{1}(M, \partial M)$ on the sutured Floer homology $S F H(M, \gamma)$. It turns out that the contact invariant $E H(M, \gamma, \xi)$ is usually sent to zero by this action. This fact allows us to refine an earlier result proved by Ghiggini and the author. As a corollary, we classify knots in $\#^{n}\left(S^{1} \times S^{2}\right)$ which have simple knot Floer homology groups: They are essentially the Borromean knots. This answers a question of Ozsváth.

In a different direction, we show that the only links in $S^{3}$ with simple knot Floer homology groups are the unlinks.
\end{abstract}

\section{Introduction}

Heegaard Floer homology, introduced by Ozsváth and Szabó [16], is a powerful theory in low-dimensional topology. In its most fundamental form, the theory constructs a chain complex $\widehat{C F}(Y)$ for each closed oriented 3manifold $Y$, such that the homology $\widehat{H F}(Y)$ of the chain complex is a topological invariant of $Y$. In [9], Juhász adapted the construction of $\widehat{H F}(Y)$ to sutured manifolds, hence defined the sutured Floer homology $\operatorname{SFH}(M, \gamma)$ for a balanced sutured manifold $(M, \gamma)$.

In [16], there is an action $A_{\zeta}$ on the Heegaard Floer homology for any $\zeta \in$ $H_{1}(Y ; \mathbb{Z}) /$ Tors, which satisfies that $A_{\zeta}^{2}=0$. This induces a $\Lambda^{*}\left(H_{1}(Y ; \mathbb{Z}) /\right.$ Tors $)-$ module structure on the Heegaard Floer homology. The goal of this paper is to define an action $A_{\zeta}$ on $\operatorname{SFH}(M, \gamma)$ for any $\zeta \in H_{1}(M, \partial M ; \mathbb{Z}) /$ Tors, hence make $S F H(M, \gamma)$ a $\Lambda^{*}\left(H_{1}(M, \partial M ; \mathbb{Z}) /\right.$ Tors $)$-module.

Juhász [10] proved that if there is a sutured manifold decomposition

$$
(M, \gamma) \stackrel{S}{\rightsquigarrow}\left(M^{\prime}, \gamma^{\prime}\right)
$$

with good properties, then $S F H\left(M^{\prime}, \gamma^{\prime}\right)$ is a direct summand of $S F H(M, \gamma)$. Hence there is an inclusion map $\iota: S F H\left(M^{\prime}, \gamma^{\prime}\right) \rightarrow S F H(M, \gamma)$ and a projection map $\pi: S F H(M, \gamma) \rightarrow S F H\left(M^{\prime}, \gamma^{\prime}\right)$ such that

$$
\pi \circ \iota=\mathrm{id}
$$


It is natural to expect that Juhász's decomposition formula respects the action of $H_{1}(M, \partial M ; \mathbb{Z}) /$ Tors. Our main theorem confirms this expectation.

Theorem 1.1. Let $(M, \gamma)$ be a balanced sutured manifold and let

$$
(M, \gamma) \stackrel{S}{\rightsquigarrow}\left(M^{\prime}, \gamma^{\prime}\right)
$$

be a well-groomed sutured manifold decomposition. Let

$$
i_{*}: H_{1}(M, \partial M) \rightarrow H_{1}(M,(\partial M) \cup S) \cong H_{1}\left(M^{\prime}, \partial M^{\prime}\right)
$$

be the map induced by the inclusion map $i:(M, \partial M) \rightarrow(M,(\partial M) \cup S)$. If $\zeta \in H_{1}(M, \partial M)$, then $i_{*}(\zeta) \in H_{1}\left(M^{\prime}, \partial M^{\prime}\right)$. Then

$$
\iota \circ A_{i_{*}(\zeta)}=A_{\zeta} \circ \iota, \quad A_{i_{*}(\zeta)} \circ \pi=\pi \circ A_{\zeta},
$$

where $\iota, \pi$ are the inclusion and projection maps defined before.

A corollary of Theorem 1.1 is the following one.

Corollary 1.2. Suppose $K$ is a nullhomologous knot in a closed oriented manifold $Y$ such that $Y-K$ is irreducible. Suppose $F$ is a Thurston norm minimizing Seifert surface for K. Let

$$
\operatorname{Ker} A=\left\{x \in \widehat{H F K}(Y, K,[F],-g) \mid A_{\zeta}(x)=0 \text { for all } \zeta \in H_{1}(Y) / \text { Tors }\right\},
$$

which is a subgroup of $\widehat{H F K}(Y, K,[F],-g)$. If $F$ is not the fiber of any fibration (if there is any) of $Y-K$, then the rank of $\operatorname{Ker} A$ is at least 2.

Ghiggini [4] and $\mathrm{Ni}$ [13] have proved that if $F$ is not the fiber of any fibration of $Y-K$, then the rank of $\overline{H F K}(Y, K,[F],-g)$ is at least 2. Since Ker $A$ is a subgroup of $\widehat{H F K}(Y, K,[F],-g)$, the above corollary can be viewed as a refinement of the theorem of Ghiggini and $\mathrm{Ni}$.

\subsection{Knots in $\#^{n}\left(S^{1} \times S^{2}\right)$ with simple knot Floer homology}

Corollary 1.2 is most useful when $\widehat{H F}(Y)$ has a rich $\Lambda^{*}\left(H_{1}(Y) /\right.$ Tors)-module structure. As an illustration, we will study knots in $\#^{n}\left(S^{1} \times S^{2}\right)$ that have simple knot Floer homology.

Suppose $K$ is a rationally null-homologous knot in $Y$, Ozsváth-Szabó $[15,19]$ and Rasmussen $[20]$ showed that $K$ specifies a filtration on $\widehat{C F}(Y)$. 
The associated homology of the filtered chain complex is the knot Floer homology $\widehat{H F K}(Y, K)$. From the construction of knot Floer homology, one sees that

$$
\operatorname{rank} \widehat{H F K}(Y, K) \geq \operatorname{rank} \widehat{H F}(Y),
$$

for any rationally null-homologous knot $K \subset Y$. When the equality holds, we say that the knot has simple knot Floer homology.

To an oriented null-homologous $n$-component link $L \subset Y$, Ozsváth and Szabó [15] associated a null-homologous knot $\kappa(L) \subset \kappa(Y)=Y \#^{n-1}\left(S^{1} \times\right.$ $S^{2}$ ), and defined the knot Floer homology of $L$ to be the knot Floer homology of $\kappa(L)$. Hence

$$
\operatorname{rank} \widehat{H F K}(Y, L) \geq \operatorname{rank} \widehat{H F}(\kappa(Y))=2^{n-1} \operatorname{rank} \widehat{H F}(Y)
$$

When the equality holds, we say that the link has simple knot Floer homology.

Clearly, the unknot in $Y$ always has simple Floer homology. Sometimes there are other knots with this property. For example, the core of a solid torus in the genus-1 Heegaard splitting of a lens space has simple Floer homology. Moreover, if two knots $\left(Y_{1}, K_{1}\right)$ and $\left(Y_{2}, K_{2}\right)$ have simple Floer homology groups, then their connected sum $\left(Y_{1} \# Y_{2}, K_{1} \# K_{2}\right)$ also has simple Floer homology. In particular, $\left(Y_{1} \# Y_{2}, K_{1}\right)$ has simple Floer homology.

It is an interesting problem to determine all null-homologous knots with simple Floer homology. For example, Hedden [5] and Rasmussen [21] showed that if a knot $L \subset S^{3}$ admits an integral lens space surgery, then the core of the surgery is a knot in the lens space with simple knot Floer homology group. Hence the classification of knots with simple Floer homology groups in lens spaces will lead to a resolution of Berge's conjecture on lens space surgery.

For certain 3-manifolds, the classification of knots with simple Floer homology groups are already known. A deep theorem of Ozsváth-Szabó [14, Theorem 1.2] implies that the only knot in $S^{3}$ with simple Floer homology group is the unknot. The author [12] generalized Ozsváth-Szabó's theorem. As a corollary, if $Y$ is an integer homology sphere which is an $L$-space, then the unknot is the only knot in $Y$ with simple Floer homology group.

We will determine all knots in $Y_{n}=\#^{n}\left(S^{1} \times S^{2}\right)$ with simple knot Floer homology. Besides the unknot, there is a class of knots called Borromean knots which have simple Floer homology groups: consider the Borromean rings. Perform 0-surgery on two components of the Borromean rings, then the third component becomes a knot in $Y_{2}$, called the Borromean knot $B_{1}$. The Borromean knot $B_{k} \subset Y_{2 k}$ is obtained by taking the connected sum 


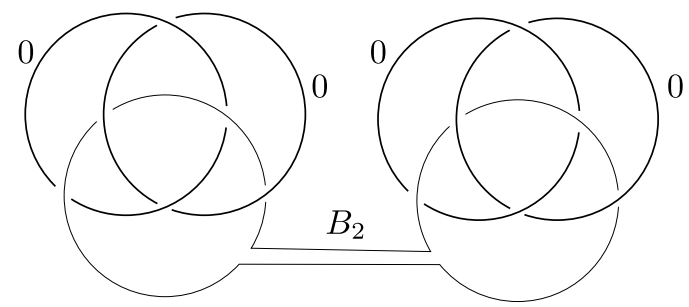

Figure 1: The Borromean knot $B_{2}$

of $k B_{1}$ 's, and $B_{0}$ is understood to be the unknot in $S^{3}$. See Figure 1 . Borromean knots are characterized by the fact that $B_{k}$ is the binding of an open book decomposition of $Y_{2 k}$, such that the page is a genus $k$ surface with one boundary component, and the monodromy is the identity map. It is known that $\widehat{H F K}\left(Y_{2 k}, B_{k}\right)$ has rank $2^{2 k}$ [15]. Ozsváth [1, Problem 1.5] asked whether Borromean knots are determined by their knot Floer homology. The following theorem answered this question affirmatively.

Theorem 1.3. Suppose $K \subset Y_{n}=\#^{n}\left(S^{1} \times S^{2}\right)$ is a null-homologous knot with simple knot Floer homology. Namely,

$$
\operatorname{rank} \widehat{H F K}\left(Y_{n}, K\right)=2^{n} .
$$

Then there exists a non-negative integer $k \leq \frac{n}{2}$ such that $\left(Y_{n}, K\right)$ is obtained from the Borromean knot $\left(Y_{2 k}, B_{k}\right)$ by taking connected sum with $Y_{n-2 k}$ in the complement of $B_{k}$.

A difference between Theorem 1.3 and the previously known classification results of simple knots is, the simple knots in $Y_{n}$ include some nontrivial knots. Such situation also appears when one tries to classify simple knots in lens spaces.

For links in $S^{3}$, it is easy to determine which ones have simple Floer homology. Our result is as follows.

Proposition 1.4. Suppose $L$ is an n-component link in $S^{3}$. If the rank of its knot Floer homology $\widehat{H F K}(L)$ is $2^{n-1}$, then $L$ is the $n$-component unlink.

Remark 1.5. It is proved by $\mathrm{Ni}[12]$ that knot Floer homology detects unlinks. However, the main result there does not imply that the rank of knot Floer homology detects unlinks. Proposition 1.4 remedies this omission. 
This paper is organized as follows. In Section 2, we will define the action on sutured Floer homology and prove Theorem 1.1. In Section 3, we will study the effect of the action on the contact invariant $E H(M, \gamma, \xi)$. Then we will prove Corollary 1.2. In Section 4 we will use Heegaard Floer homology and combinatorial group theory to determine all knots in $Y_{n}$ that have simple Floer homology. In Section 5, we will show the unlinks are the only links in $S^{3}$ that have simple Floer homology.

\section{Sutured Floer homology and the homological action}

In this section, we will define the homological action on the sutured Floer homology and study its behavior under sutured manifold decompositions. We will assume the readers have some familiarity with sutured manifold theory and sutured Floer homology.

\subsection{The definition of the action}

Definition 2.1. A sutured manifold $(M, \gamma)$ is a compact oriented 3-manifold $M$ together with a set $\gamma \subset \partial M$ of pairwise disjoint annuli. The core of each component of $\gamma$ is a suture, and the set of sutures is denoted by $s(\gamma)$.

Every component of $R(\gamma)=\partial M-\operatorname{int}(\gamma)$ is oriented. Define $R_{+}(\gamma)$ (or $R_{-}(\gamma)$ ) to be the union of those components of $R(\gamma)$ whose normal vectors point out of (or into) $M$. The orientations on $R(\gamma)$ must be coherent with respect to $s(\gamma)$.

A balanced sutured manifold is a sutured manifold $(M, \gamma)$ without closed components, such that $\chi\left(R_{+}(\gamma)\right)=\chi\left(R_{-}(\gamma)\right)$, and $\gamma$ intersects each component of $\partial M$.

Let $(M, \gamma)$ be a sutured manifold, and $S$ a properly embedded surface in M. According to Gabai [2], there is a natural way to put a sutured manifold structure on $M^{\prime}=M \backslash \nu(S)$. This process is a sutured manifold decomposition

$$
(M, \gamma) \stackrel{S}{\rightsquigarrow}\left(M^{\prime}, \gamma^{\prime}\right)
$$

Definition 2.2. A sutured manifold decomposition is well-groomed, if for every component $V$ of $R(\gamma), V \cap S$ is a union of parallel oriented nonseparating simple closed curves or arcs. 
Definition 2.3. A sutured manifold hierarchy is a sequence of decompositions

$$
\left(M_{0}, \gamma_{0}\right) \stackrel{S_{0}}{\rightsquigarrow}\left(M_{1}, \gamma_{1}\right) \stackrel{S_{1}}{\rightsquigarrow}\left(M_{2}, \gamma_{2}\right) \stackrel{S_{2}}{\rightsquigarrow} \ldots \stackrel{S_{n-1}}{\rightsquigarrow}\left(M_{n}, \gamma_{n}\right)
$$

such that $\left(M_{n}, \gamma_{n}\right)$ is a disjoint union of $\left(D^{2} \times I,\left(\partial D^{2}\right) \times I\right)$ 's.

A fundamental theorem of Gabai [2] says that for any taut sutured manifold, there exists a well-groomed sutured manifold hierarchy.

Suppose $(\Sigma, \boldsymbol{\alpha}, \boldsymbol{\beta})$ is an admissible sutured Heegaard diagram for a balanced sutured manifold $(M, \gamma)$. Let $\omega$ be a relative $1-$ cycle on $\Sigma$, such that it is in general position with the $\alpha$ - and $\beta$-curves. Namely, $\omega=\sum k_{i} c_{i}$, where $k_{i} \in \mathbb{Z}$, each $c_{i}$ is a properly immersed oriented curve on $\Sigma$, such that $c_{i}$ is transverse to $\alpha$ - and $\beta$-curves, and $c_{i}$ does not contain any intersection point of $\alpha$ - and $\beta$-curves.

We can also regard $\omega$ as a relative 1 -cycle representing a class in $H_{1}(M, \partial M)$. On the other hand, any homology class in $H_{1}(M, \partial M)$ can be represented by a relative 1-cycle on $\Sigma$, since the maps

$$
H_{1}(\Sigma, \partial \Sigma) \rightarrow H_{1}(M, \gamma), \quad H_{1}(M, \gamma) \rightarrow H_{1}(M, \partial M)
$$

are both surjective.

Let $\mathbf{x}, \mathbf{y} \in \mathbb{T}_{\alpha} \cap \mathbb{T}_{\beta}$. If $\phi$ is a topological Whitney disc connecting $\mathbf{x}$ to $\mathbf{y}$, let $\partial_{\alpha} \phi=(\partial \phi) \cap \mathbb{T}_{\alpha}$. We can also regard $\partial_{\alpha} \phi$ as a multi-arc that lies on $\Sigma$ and connects $\mathbf{x}$ to $\mathbf{y}$. Similarly, we define $\partial_{\beta} \phi$ as a multi-arc connecting $\mathbf{y}$ to $\mathbf{x}$. We define

$$
a(\omega, \phi)=\# \widehat{\mathcal{M}}(\phi)\left(\omega \cdot\left(\partial_{\alpha} \phi\right)\right)
$$

where $\omega \cdot\left(\partial_{\alpha} \phi\right)$ is the algebraic intersection number of $\omega$ and $\partial_{\alpha} \phi$. Let

$$
A_{\omega}: \operatorname{SFC}(M, \gamma) \rightarrow \operatorname{SFC}(M, \gamma)
$$

be defined as

$$
A_{\omega}(\mathbf{x})=\sum_{\mathbf{y} \in \mathbb{T}_{\alpha} \cap \mathbb{T}_{\beta}} \sum_{\left\{\phi \in \pi_{2}(\mathbf{x}, \mathbf{y}) \mid \mu(\phi)=1\right\}} a(\omega, \phi) \mathbf{y} .
$$

As in [16, Lemma 4.18], $A_{\omega}$ is a chain map. The following lemma shows that it induces a well defined action of $H_{1}(M, \partial M ; \mathbb{Z}) /$ Tors on $\operatorname{SFH}(M, \gamma)$.

Lemma 2.4. Suppose $\omega_{1}, \omega_{2} \subset \Sigma$ are two relative 1-cycles which are homologous in $H_{1}(M, \partial M ; \mathbb{Z}) /$ Tors, then $A_{\omega_{1}}$ is chain homotopic to $A_{\omega_{2}}$. 
Proof. Since $\omega_{1}$ and $\omega_{2}$ are homologous in $H_{1}(M, \partial M ; \mathbb{Z}) /$ Tors, there exists a nonzero integer $m$ such that $m\left[\omega_{1}\right]=m\left[\omega_{2}\right] \in H_{1}(M, \partial M ; \mathbb{Z})$.

Claim. There exists a relative 2-chain $B$ in $(\Sigma, \partial \Sigma)$, such that $(\partial B) \backslash(\partial \Sigma)$ consists of $m \omega_{2}, m\left(-\omega_{1}\right)$, copies of $\alpha$-curves and $\beta$-curves, and proper curves $\xi, \eta \subset \Sigma$ such that $\xi$ is disjoint from $\alpha$-curves and $\eta$ is disjoint from $\beta$-curves.

Consider the triple $(M, \partial M, \gamma)$, we get an exact sequence

$$
H_{1}(\partial M, \gamma) \rightarrow H_{1}(M, \gamma) \rightarrow H_{1}(M, \partial M) \rightarrow 0
$$

As a consequence, if $m\left[\omega_{1}\right]=m\left[\omega_{2}\right] \in H_{1}(M, \partial M)$, then there exists an element $c \in H_{1}(\partial M, \gamma) \cong H_{1}(R(\gamma), \partial R(\gamma))$, such that $c+m\left[\omega_{2}\right]-m\left[\omega_{1}\right]$ is homologous to zero in $H_{1}(M, \gamma)$. Let $\xi^{\prime} \subset R_{-}(\gamma), \eta^{\prime} \subset R_{+}(\gamma)$ be proper curves such that $\xi^{\prime}+\eta^{\prime}$ represents $c \in H_{1}(R(\gamma), \partial R(\gamma))$. Using the gradient flow of a Morse function associated with the sutured diagram, we can project $\xi^{\prime}, \eta^{\prime}$ to proper curves $\xi, \eta \subset \Sigma$ such that $\xi$ is disjoint from $\alpha$-curves and $\eta$ is disjoint from $\beta$-curves. Then $\xi+\eta+m\left[\omega_{2}\right]-m\left[\omega_{1}\right]$ is homologous to zero in $H_{1}(M, \gamma)$. Using the fact that

$$
H_{1}(M, \gamma) \cong H_{1}(\Sigma, \partial \Sigma) /\left(\left[\alpha_{1}\right] \ldots,\left[\alpha_{g}\right],\left[\beta_{1}\right], \ldots,\left[\beta_{g}\right]\right)
$$

we conclude that there is a relative 2 -chain $B$ in $(\Sigma, \partial \Sigma)$, such that $(\partial B) \backslash(\partial \Sigma)$ consists of $m \omega_{2}, m\left(-\omega_{1}\right), \xi, \eta$, and copies of $\alpha$-curves and $\beta$-curves. This finishes the proof of the claim.

Perturbing $B$ slightly, we get a 2 -chain $B^{\prime}$ such that

$$
\left(\partial B^{\prime}\right) \backslash(\partial \Sigma)=m \omega_{2}-m \omega_{1}+\sum\left(a_{i} \alpha_{i}^{\prime}+b_{i} \beta_{i}^{\prime}\right)+\xi+\eta,
$$

where $\alpha_{i}^{\prime}, \beta_{i}^{\prime}$ are parallel copies of $\alpha_{i}, \beta_{i}$.

Let $\phi$ be a topological Whitney disc (or more generally, a higher genus surface) connecting $\mathbf{x}$ to $\mathbf{y}$. Since $\alpha_{i}^{\prime}, \xi$ are disjoint from all $\alpha$-curves, we have $\alpha_{i}^{\prime} \cdot \partial_{\alpha} \phi=\xi \cdot \partial_{\alpha} \phi=0$. Similarly,

$$
\beta_{i}^{\prime} \cdot \partial_{\alpha} \phi=-\beta_{i}^{\prime} \cdot \partial_{\beta} \phi=0, \quad \eta \cdot \partial_{\alpha} \phi=0 .
$$

We have

$$
n_{\mathbf{x}}\left(B^{\prime}\right)-n_{\mathbf{y}}\left(B^{\prime}\right)=-\left(\left(\partial B^{\prime}\right) \backslash(\partial \Sigma)\right) \cdot \partial_{\alpha} \phi=m\left(\omega_{1}-\omega_{2}\right) \cdot \partial_{\alpha} \phi \in m \mathbb{Z} .
$$

Pick an intersection point $\mathbf{x}_{0}$, and let $\mathfrak{s}$ by the relative $\operatorname{Spin}^{c}$ structure represented by $\mathbf{x}_{0}$. After adding copies of $\Sigma$ to $B^{\prime}$, we can assume that $n_{\mathbf{x}_{0}}\left(B^{\prime}\right)$ is divisible by $m$. Since any two intersection points representing $\mathfrak{s}$ 
are connected by a topological Whitney disc or higher genus surface, (1) implies that $n_{\mathbf{x}}\left(B^{\prime}\right)$ is divisible by $m$ for any $\mathbf{x}$ representing $\mathfrak{s}$.

Now we define a map $H: S F C(M, \gamma, \mathfrak{s}) \rightarrow \operatorname{SFC}(M, \gamma, \mathfrak{s})$ by letting

$$
H(\mathbf{x})=\frac{n_{\mathbf{x}}\left(B^{\prime}\right)}{m} \mathbf{x}
$$

It follows from (1) that

$$
A_{\omega_{1}}-A_{\omega_{2}}=\partial \circ H-H \circ \partial .
$$

Namely, $A_{\omega_{1}}, A_{\omega_{2}}$ are chain homotopic.

Now the same argument as in [16, Lemma 4.17] shows that $A_{\zeta}$ is a differential for any $\zeta \in H_{1}(M, \partial M) /$ Tors, hence $A$ gives rise to an action of $\Lambda^{*}\left(H_{1}(M, \partial M) /\right.$ Tors $)$ on $S F H(M, \gamma)$.

\subsection{Sutured manifold decomposition}

We recall a few basic notations about sutured manifolds from $[2,3,9,10]$.

Suppose

$$
(M, \gamma) \stackrel{S}{\rightsquigarrow}\left(M^{\prime}, \gamma^{\prime}\right)
$$

is a well-groomed sutured manifold decomposition. In [10], Juhász constructed a surface diagram

$$
(\Sigma, \boldsymbol{\alpha}, \boldsymbol{\beta}, P)
$$

adapted to $S$, where $P \subset \Sigma$ is a compact surface with corners such that $P \cap(\partial \Sigma)$ consists of exactly the vertices of $P$. Moreover,

$$
\partial P=A \cup B
$$

where $A, B$ are unions of edges of $P$ with $A \cap B=P \cap(\partial \Sigma), A \cap \boldsymbol{\beta}=\emptyset, B \cap$ $\boldsymbol{\alpha}=\emptyset$. $(\Sigma, \boldsymbol{\alpha}, \boldsymbol{\beta})$ is a balanced diagram for $(M, \gamma)$. An admissible balanced diagram

$$
\left(\Sigma^{\prime}, \boldsymbol{\alpha}^{\prime}, \boldsymbol{\beta}^{\prime}\right)
$$

for $\left(M^{\prime}, \gamma^{\prime}\right)$ can be constructed as follows:

$$
\Sigma^{\prime}=(\Sigma \backslash P) \cup P_{A} \cup P_{B},
$$


where $P_{A}, P_{B}$ are two copies of $P$, and $\Sigma \backslash P$ is glued to $P_{A}$ along $A$ while glued to $P_{B}$ along $B$. There is a natural projection map $p: \Sigma^{\prime} \rightarrow \Sigma$, and

$$
\boldsymbol{\alpha}^{\prime}=p^{-1}(\boldsymbol{\alpha}) \backslash P_{B}, \quad \boldsymbol{\beta}^{\prime}=p^{-1}(\boldsymbol{\beta}) \backslash P_{A} .
$$

The decomposing surface $S$ can be seen from the surface diagram as follows: $M$ can be obtained from $\Sigma \times[0,1]$ by adding 2-handles along $\alpha_{i} \times 0$ 's and $\beta_{j} \times 1$ 's. Then $S \subset M$ is isotopic to the surface

$$
\left(P \times \frac{1}{2}\right) \cup\left(A \times\left[\frac{1}{2}, 1\right]\right) \cup\left(B \times\left[0, \frac{1}{2}\right]\right) .
$$

Let $O_{P}$ be the set of intersection points in $\mathbb{T}_{\alpha} \cap \mathbb{T}_{\beta}$ that are supported outside of $P$. Then $O_{P}$ consists of the points whose associated relative $\operatorname{Spin}^{c}$ structures are "extremal" with respect to $S$. And $O_{P}$ is in one-to-one correspondence with $\mathbb{T}_{\alpha^{\prime}} \cap \mathbb{T}_{\beta^{\prime}}$.

Using techniques introduced by Sarkar and Wang [22], Juhász proved that the surface diagram can be made "nice". In particular, if $\phi$ is a holomorphic disc connecting two points in $O_{P}$ with $\mu(\phi)=1$, then the domain of $\phi$ is either an embedded bigon or square. Moreover, the following fact was contained in the proof of [10, Proposition 7.6].

Lemma 2.5. Suppose $\mathcal{D}$ is the domain of a holomorphic disc connecting two points in $O_{P}$ with $\mu(\phi)=1$, and $C$ is a component of $\mathcal{D} \cap P$. Then $C$ is either a bigon or a square. If $C$ is a bigon, then $C$ has either an $\alpha$-edge and an $A$-edge, or a $\beta$-edge and a $B$-edge. If $C$ is a square, then $C$ has either two opposite $\alpha$-edges and two opposite $A$-edges, or two opposite $\beta$-edges and two opposite $B$-edges.

Lemma 2.6. Suppose $\zeta \in H_{1}(M, \partial M)$, then $\zeta$ can be represented by a relative 1 -cycle $\omega \subset \Sigma$, such that $\omega$ intersects $\partial P$ in the interior of $A$. As a result, $\omega$ can be lifted to a relative $1-$ cycle $\omega^{\prime} \subset\left((\Sigma \backslash P) \cup_{A} P_{A}\right) \subset$ $\Sigma^{\prime}$, such that $p$ maps $\omega^{\prime}$ homeomorphically to $\omega$, and $\omega^{\prime}$ represents $i_{*}(\zeta) \in$ $H_{1}\left(M^{\prime}, \partial M^{\prime}\right)$.

Proof. Let $\omega_{0} \subset \Sigma$ be a relative 1-cycle representing $\zeta$, such that $\omega_{0}$ intersects $\partial P$ transversely in the interior of the edges. Suppose $\omega_{0}$ has an intersection point with $B$. After homotoping $\omega_{0}$, we may assume this intersection point is near a corner of $P$. As in Figure 2, we can replace $\omega_{0}$ by a new relative $1-$ cycle $\omega_{1}$, such that $\left[\omega_{1}\right]=\left[\omega_{0}\right] \in H_{1}(\Sigma, \partial \Sigma)$, and $\#\left(\omega_{1} \cap B\right)=$ $\#\left(\omega_{0} \cap B\right)-1$. Continuing this process, we get a relative 1-cycle $\omega$ representing $\zeta$, which does not intersect $B$. 

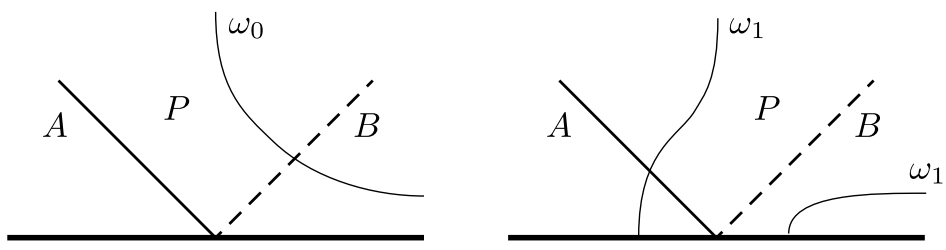

Figure 2: Changing $\omega_{0}$ near a corner of $P$

Cutting $\Sigma$ open along $B$, we get a surface homeomorphic to $(\Sigma \backslash P) \cup_{A}$ $P_{A}$. Since $\omega$ does not intersect $B$, it lies in the new surface. Hence there is a corresponding relative 1-cycle $\omega^{\prime} \subset(\Sigma \backslash P) \cup_{A} P_{A}$, such that $p$ maps $\omega^{\prime}$ homeomorphically to $\omega$.

$S$ is isotopic to a surface (2). Since $\omega$ is disjoint from $B, \omega \times\left(\frac{1}{2}-\epsilon\right)$ is disjoint from $S$. Cutting $M$ open along $S, \omega \times\left(\frac{1}{2}-\epsilon\right)$ becomes the relative 1 -cycle $\omega^{\prime} \times\left(\frac{1}{2}-\epsilon\right)$. Hence $\omega^{\prime}$ represents $i_{*}(\zeta)$ in $H_{1}\left(M^{\prime}, \partial M^{\prime}\right)$.

Now we are ready to prove Theorem 1.1.

Proof of Theorem 1.1. The projection map $p: \Sigma^{\prime} \rightarrow \Sigma$ induces a bijection

$$
p_{*}: \mathbb{T}_{\alpha^{\prime}} \cap \mathbb{T}_{\beta^{\prime}} \rightarrow O_{P},
$$

which then induces the inclusion map

$$
S F C\left(M^{\prime}, \gamma^{\prime}\right) \rightarrow S F C(M, \gamma) .
$$

As argued in [10], $p$ induces a one-to-one correspondence between the holomorphic discs for $\operatorname{SFC}\left(M^{\prime}, \gamma^{\prime}\right)$ and the holomorphic discs for the chain complex generated by $O_{P}$. Let $\omega$ be the curve obtained in Lemma 2.6. Suppose $\phi$ is a holomorphic disc connecting two points in $O_{P}, \phi^{\prime}$ is the corresponding holomorphic disc connecting two points in $\mathbb{T}_{\alpha^{\prime}} \cap \mathbb{T}_{\beta^{\prime}}$. The intersection points of $\partial_{\alpha} \phi$ and $\omega$ outside of $P$ are in one-to-one correspondence with the intersection points of $\partial_{\alpha} \phi^{\prime}$ and $\omega^{\prime}$ outside of $P_{A} \cup P_{B}$.

Let $C$ be a component of $\mathcal{D} \cap P$, where $\mathcal{D}$ is the domain of $\phi$. Let $C^{\prime}$ be the corresponding component of $\mathcal{D}^{\prime} \cap\left(P_{A} \cup P_{B}\right)$. By Lemma 2.5, $C \cap(\partial P)$ is contained in either $A$ or $B$. If $(C \cap(\partial P)) \subset A$, then $C^{\prime} \subset P_{A}$, and $\left(\partial_{\alpha} C\right) \cap \omega$ is in one-to-one correspondence with $\left(\partial_{\alpha} C^{\prime}\right) \cap \omega^{\prime}$. If $(C \cap(\partial P)) \subset B$, then $C^{\prime} \subset P_{B}$, so $\left(\partial_{\alpha} C^{\prime}\right) \cap \omega^{\prime}=\emptyset$. By Lemma 2.5 , in this case $C$ has no $\alpha$-edge, 
so $\left(\partial_{\alpha} C\right) \cap \omega=\emptyset$. This shows that

$$
\omega \cdot\left(\partial_{\alpha} \phi\right)=\omega^{\prime} \cdot\left(\partial_{\alpha} \phi^{\prime}\right) .
$$

Now our desired result follows from the definition of the homological action.

\subsection{The homological action on Knot Floer homology}

The material in this subsection is not used in this paper. However, it is helpful to have in mind the symmetry stated in Proposition 2.7.

Suppose $K$ is a null-homologous knot in a closed 3-manifold $Y$. As in [15], let

$$
(\Sigma, \boldsymbol{\alpha}, \boldsymbol{\beta}, w, z)
$$

be a doubly pointed Heegaard diagram for $(Y, K)$ which is induced from a marked Heegaard diagram

$$
\left(\Sigma, \boldsymbol{\alpha}, \boldsymbol{\beta}_{0}, \mu, m\right)
$$

Fix a $\operatorname{Spin}^{c}$ structure $\mathfrak{s}$ on $Y$ and let $\mathfrak{t} \in \operatorname{Spin}^{c}(Y, K) \cong \operatorname{Spin}^{c}\left(Y_{0}(K)\right)$ be a relative $\operatorname{Spin}^{c}$ structure which extends it. Let $C F K^{\infty}(Y, K, \mathfrak{t})$ be an abelian group freely generated by triples $[\mathbf{x}, i, j]$ with

$$
\mathbf{x} \in \mathbb{T}_{\alpha} \cap \mathbb{T}_{\beta}, \quad \mathfrak{s}_{w}(\mathbf{x})=\mathfrak{s}
$$

and

$$
\underline{\mathfrak{s}}_{m}(\mathbf{x})+(i-j) P D[\mu]=\mathfrak{t},
$$

where $\underline{\mathfrak{s}}_{m}: \mathbb{T}_{\alpha} \cap \mathbb{T}_{\alpha} \rightarrow \operatorname{Spin}^{c}(Y, K)$ is the map defined in [15]. The chain complex is endowed with the differential

$$
\partial^{\infty}[\mathbf{x}, i, j]=\sum_{\mathbf{y} \in \mathbb{T}_{\alpha} \cap \mathbb{T}_{\beta}} \sum_{\left\{\phi \in \pi_{2}(\mathbf{x}, \mathbf{y}) \mid \mu(\phi)=1\right\}} \#(\widehat{\mathcal{M}}(\phi))\left[\mathbf{y}, i-n_{w}(\phi), j-n_{z}(\phi)\right] .
$$

The homology of $\left(C F K^{\infty}(Y, K, \mathfrak{t}), \partial^{\infty}\right)$ is denoted $H F K^{\infty}(Y, K, \mathfrak{t})$.

Suppose $\omega$ is a 1-cycle on $\Sigma$. Let

$$
A_{\omega}[\mathbf{x}, i, j]=\sum_{\mathbf{y} \in \mathbb{T}_{\alpha} \cap \mathbb{T}_{\beta}} \sum_{\left\{\phi \in \pi_{2}(\mathbf{x}, \mathbf{y}) \mid \mu(\phi)=1\right\}} a(\omega, \phi)\left[\mathbf{y}, i-n_{w}(\phi), j-n_{z}(\phi)\right] .
$$

As in $[16]$ and the arguments before, $A_{\omega}$ induces an action of $\Lambda^{*} H_{1}(Y ; \mathbb{Z}) /$ Tors on $H_{F} K^{\infty}(Y, K, \mathfrak{t})$. 
There is a $U$-action on $C F K^{\infty}(Y, K, \mathfrak{t})$ given by

$$
U[\mathbf{x}, i, j]=[\mathbf{x}, i-1, j-1] .
$$

Let $C F K^{-, *}(Y, K, \mathfrak{t})$ be the subcomplex of $C F K^{\infty}(Y, K, \mathfrak{t})$ generated by $[\mathbf{x}, i, j]$ with $i<0$, and let $C F K^{+, *}(Y, K, \mathfrak{t})$ be its quotient complex. Moreover, let

$$
C F K^{0, *}(Y, K, \mathfrak{t}) \subset C F K^{+, *}(Y, K, \mathfrak{t})
$$

be the kernel of the induced $U$-action. There is a filtration on $C F K^{0, *}(Y, K, \mathfrak{t})$ given by the grading $j$, and the associated graded complex is denoted $\overline{C F K}(Y, K, \mathfrak{t})$. There are induced actions of $A_{\omega}$ on the above complexes, and the actions induce differentials $A_{[\omega]}$ on the corresponding homology groups.

When $\mathfrak{s}$ is a torsion $\operatorname{Spin}^{c}$ structure over $Y$, as in Ozsváth and Szabó [15] there is an absolute $\mathbb{Q}$-grading on $C F K^{\infty}(Y, K, \mathfrak{t})$ and the induced complexes. Let $\widehat{H F K}_{d}(Y, K, \mathfrak{t})$ be the summand of $\widehat{H F K}(Y, K, \mathfrak{t})$ at the absolute grading $d$.

Proposition 2.7. Let $\mathfrak{s}$ be a torsion Spin ${ }^{c}$ structure over $Y$, and let $\mathfrak{t} \in$ $\operatorname{Spin}^{c}(Y, K) \cong \operatorname{Spin}^{c}\left(Y_{0}(K)\right)$ be a relative Spin ${ }^{c}$ structure which extends $\mathfrak{s}$. $\overline{\text { Let } \zeta}$ be a homology class in $H_{1}(Y ; \mathbb{Z}) /$ Tors. Then there is an isomorphism

$$
f: \widehat{H F K}_{d}(Y, K, \mathfrak{t}) \stackrel{\cong}{\longrightarrow} \widehat{H F K}_{d-2 m}(Y, K, J \mathfrak{t}),
$$

such that the following diagram is commutative:

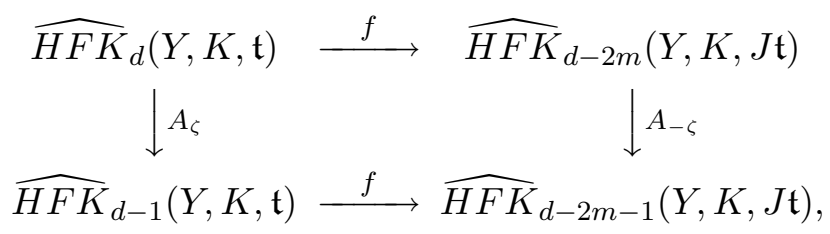

where $2 m=\left\langle c_{1}(\mathfrak{t}),[\widehat{F}]\right\rangle$ for any $\widehat{F} \subset Y_{0}(K)$ which is obtained by capping off the boundary of a Seifert surface $F$ for $K$ with a disc.

Proof. The existence of the isomorphism $f$ is already proved in Ozsváth and Szabó [15]. We will follow their argument to prove the commutative diagram. 
Let

$$
\Gamma_{1}=(\Sigma, \boldsymbol{\alpha}, \boldsymbol{\beta}, w, z)
$$

be a doubly pointed Heegaard diagram for $(Y, K)$. Then

$$
\Gamma_{2}=(-\Sigma, \boldsymbol{\beta}, \boldsymbol{\alpha}, z, w)
$$

is also a Heegaard diagram for $(Y, K)$. If $\mathbf{x} \in \mathbb{T}_{\alpha} \cap \mathbb{T}_{\beta}$ represents $\mathfrak{s}$ in $\Gamma_{1}$, then $\mathbf{x}$ represents $J \mathfrak{s}$ in $\Gamma_{2}$. If $\phi$ is a holomorphic disc in $\Gamma_{1}$ connecting $\mathbf{x}$ to $\mathbf{y}$, then $\phi$ gives rise to a holomorphic $\operatorname{disc} \bar{\phi}$ in $\Gamma_{2}$ connecting $\mathbf{x}$ to $\mathbf{y}$. Topologically, $\bar{\phi}$ is just $-\phi$. Let $\omega \subset \Sigma$ be a curve representing $\zeta$, then

$$
\left.\omega \cdot \partial_{\alpha} \phi\right|_{\Sigma}=-\left.\omega \cdot \partial_{\beta} \phi\right|_{\Sigma}=-\left.\omega \cdot \partial_{\beta} \bar{\phi}\right|_{-\Sigma}
$$

where the notation $\left.\right|_{\Sigma}$ or $\left.\right|_{-\Sigma}$ implies that the intersection number is evaluated in $\Sigma$ or $-\Sigma$.

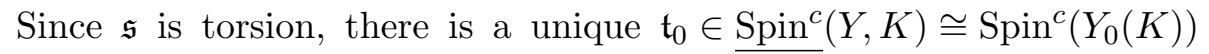
extending $\mathfrak{s}$ which satisfies

$$
\left\langle c_{1}\left(\mathfrak{t}_{0}\right),[\widehat{F}]\right\rangle=0
$$

for any Seifert surface $F$ for $K$. Using the observations in the first paragraph, it follows that if we interchange the roles of $i$ and $j$, then the chain complex $C F K^{\infty}\left(Y, K, \mathfrak{t}_{0}\right)$ can be viewed as the chain complex $C F K^{\infty}\left(Y, K, J \mathfrak{t}_{0}\right)$. It follows that there is a grading preserving isomorphism

$$
C F K^{-m, 0}\left(Y, K, \mathfrak{t}_{0}\right) \cong C F K^{0,-m}\left(Y, K, J \mathfrak{t}_{0}\right) \cong \widehat{C F K}(Y, K, J \mathfrak{t})
$$

Moreover, the map $U^{m}$ induces an isomorphism

$$
U^{m}: \widehat{C F K}(Y, K, \mathfrak{t})=C F K^{0, m}\left(Y, K, \mathfrak{t}_{0}\right) \rightarrow C F K^{-m, 0}\left(Y, K, \mathfrak{t}_{0}\right)
$$

which decreases the absolute grading by $2 m$. Hence

$$
\widehat{C F K}_{d}(Y, K, \mathfrak{t}) \cong \widehat{C F K}_{d-2 m}(Y, K, J \mathfrak{t}) .
$$

Using (3), we find that the action of $A_{\omega}$ on $\widehat{C F K}_{d}(Y, K, \mathfrak{t})$ corresponds to the action of $A_{-\omega}$ on $\widehat{C F K}_{d-2 m}(Y, K, J \mathfrak{t})$. 


\section{The contact invariant $\boldsymbol{E H}(M, \gamma, \xi)$}

Suppose $(M, \gamma)$ is a sutured manifold. A contact structure on $(M, \gamma)$ is a contact structure $\xi$ on $M$, such that $\partial M$ is convex and the suture $s(\gamma)$ is the dividing set. Suppose

$$
(M, \gamma) \stackrel{S}{\rightsquigarrow}\left(M^{\prime}, \gamma^{\prime}\right)
$$

is a taut decomposition, and $\xi$ is a contact structure on $(M, \gamma)$ such that $S$ is convex and the dividing set $\gamma_{S}$ on $S$ is $\partial$-parallel, namely, each component of $\gamma_{S}$ cuts off a disc containing no other component of $\gamma_{S}$. Let $\xi^{\prime}$ be the restriction of $\xi$ on $\left(M^{\prime}, \gamma^{\prime}\right)$. Then $\xi^{\prime}$ is tight if and only if $\xi$ is tight [7].

Definition 3.1. Suppose $(M, \gamma)$ is a taut sutured manifold. A tight contact structure $\xi$ on $(M, \gamma)$ is of hierarchy type, if there exists a well-groomed sutured manifold hierarchy (see Definitions 2.2 and 2.3)

$$
(M, \gamma)=\left(M_{0}, \gamma_{0}\right) \stackrel{S_{0}}{\rightsquigarrow}\left(M_{1}, \gamma_{1}\right) \stackrel{S_{1}}{\rightsquigarrow}\left(M_{2}, \gamma_{2}\right) \stackrel{S_{2}}{\rightsquigarrow} \ldots \stackrel{S_{n-1}}{\rightsquigarrow}\left(M_{n}, \gamma_{n}\right),
$$

such that the dividing set on each $S_{i}$ is $\partial$-parallel. In fact, since $M_{n}$ consists of balls, $\xi$ is obtained by gluing the unique tight contact structure on $M_{n}$ along the decomposing surfaces.

For a contact structure $\xi$ on $(M, \gamma)$, Honda et al. [8] defined an invariant $E H(M, \gamma, \xi) \in S F H(-M,-\gamma) /( \pm 1)$. They also studied the behavior of this invariant under sutured manifold decomposition.

Theorem 3.2 (Honda-Kazez-Matić). Let $(M, \gamma, \xi)$ be the contact structure obtained from $\left(M^{\prime}, \gamma^{\prime}, \xi^{\prime}\right)$ by gluing along a $\partial$-parallel $\left(S, \gamma_{S}\right)$. Under the inclusion

$$
\operatorname{SFH}\left(-M^{\prime},-\gamma^{\prime}\right) \subset S F H(-M,-\gamma)
$$

as a direct summand, $E H\left(M^{\prime}, \gamma^{\prime}, \xi^{\prime}\right)$ is mapped to $E H(M, \gamma, \xi)$.

Now Theorem 1.1 immediately implies the following result.

Corollary 3.3. Suppose $(M, \gamma)$ is a taut sutured manifold and $\xi$ is a contact structure of hierarchy type on $M$. Then for any $\zeta \in H_{1}(M, \partial M ; \mathbb{Z}) /$ Tors, $\operatorname{EH}(M, \gamma, \xi)$ lies in the kernel of $A_{\zeta}$ while $\operatorname{EH}(M, \gamma, \xi)$ is not contained in the image of $A_{\zeta}$. 
Proof. Consider a hierarchy (4) associated with the contact structure $\xi$. By Theorem 3.2, there are maps

$$
\iota: S F H\left(-M_{n},-\gamma_{n}\right) \cong \mathbb{Z} \rightarrow S F H(-M,-\gamma)
$$

which sends a generator of $\operatorname{SFH}\left(-M_{n},-\gamma_{n}\right)$ to $E H(M, \gamma, \xi)$, and

$$
\pi: S F H(-M,-\gamma) \rightarrow S F H\left(-M_{n},-\gamma_{n}\right) \cong \mathbb{Z}
$$

which sends $E H(M, \gamma, \xi)$ to a generator of $S F H\left(-M_{n},-\gamma_{n}\right)$.

Given $\zeta \in H_{1}(M, \partial M)$, let $i_{*}(\zeta)$ be its image in $H_{1}\left(M_{n}, \partial M_{n}\right)$. Since $M_{n}$ consists of balls, $i_{*}(\zeta)=0$, hence $A_{i_{*}(\zeta)}=0$. (This result also follows from the fact that $A_{i_{*}(\zeta)}$ is a differential or the fact that $A_{i_{*}(\zeta)}$ decreases the $\mathbb{Z} / 2 \mathbb{Z}$ homological grading by 1 .) Using Theorem 1.1 , we get a commutative diagram:

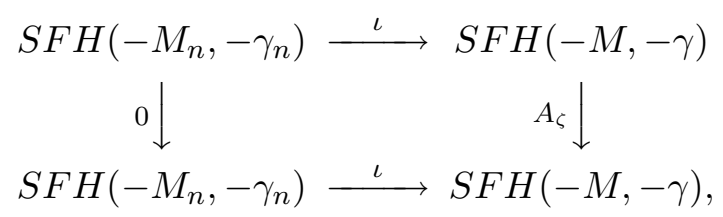

hence $A_{\zeta}(E H(M, \gamma, \xi))=0$.

Similarly, considering the commutative diagram

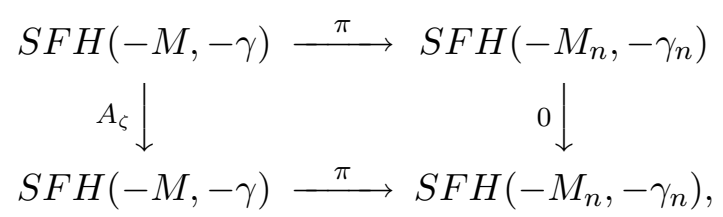

we conclude that $E H(M, \gamma, \xi)$ does not lie in the image of $A_{\zeta}$.

Remark 3.4. Since $A_{\zeta}^{2}=0, A_{\zeta}$ can be viewed as a differential on the Floer homology group, thus one can talk about its homology. Corollary 3.3 says that the contact invariant represents a nontrivial class in the homology of $A_{\zeta}$. A version of Corollary 3.3 for weakly fillable contact structures on closed manifolds was proved in [6], following the strategy of Ozsváth and Szabó [14].

Proof sketch of Corollary 1.2. Decomposing $Y-K$ along $F$, we get a taut sutured manifold $(M, \gamma)$. If $F$ is not a fiber of any fibration, then $(M, \gamma)$ is not a product sutured manifold. The argument in $[4,13]$ shows that we can construct two different sutured manifold hierarchies, and corresponding two tight contact structures $\xi_{1}, \xi_{2}$ obtained from gluing the tight contact structure on the balls via the two hierarchies, such that $E H\left(M, \gamma, \xi_{1}\right)$ and 
$E H\left(M, \gamma, \xi_{2}\right)$ are linearly independent. See also [10] for the version of argument adapted to sutured Floer homology.

It is showed in [10] that the inclusion map

$$
\operatorname{SFH}(M, \gamma) \rightarrow H F K(Y, K)
$$

induced by the decomposition

$$
Y-K \stackrel{F}{\rightsquigarrow}(M, \gamma)
$$

maps $\operatorname{SFH}(M, \gamma)$ isomorphically onto $\operatorname{HFK}(Y, K,[F],-g)$. Using Theorem 1.1 and Corollary 3.3, we conclude that Ker $A$ has rank at least 2.

The reader may find that the use of the contact class $E H$ is not necessary for the proof of Corollary 1.2. We choose this presentation so that the nontrivial elements in $\operatorname{Ker} A$ have their geometric meaning.

\section{Knots in $\#^{n} S^{1} \times S^{2}$}

Let $Y_{n}=\#^{n} S^{1} \times S^{2}, V_{n}=H_{1}\left(Y_{n} ; \mathbb{Z}\right), V_{n}^{\prime}=H^{1}\left(Y_{n} ; \mathbb{Z}\right)$. It is well known that $\widehat{H F}\left(Y_{n}\right)$ as a $\Lambda^{*} V_{n}$-module is isomorphic to $\Lambda^{*} V_{n}^{\prime}$. Namely, $\widehat{H F}\left(Y_{n}\right) \cong \Lambda^{*} V_{n}^{\prime}$ as a group, and $A_{\zeta}$ is given by the contraction homomorphism

$$
\iota_{\zeta}: \Lambda^{i} V_{n}^{\prime} \rightarrow \Lambda^{i-1} V_{n}^{\prime}
$$

Lemma 4.1. In the module $\Lambda^{*} V_{n}^{\prime}$, we have

$$
\bigcap_{\zeta \in V_{n}} \operatorname{ker} \iota_{\zeta}=\mathbb{Z} \mathbf{1}
$$

the subgroup generated by the unit element $\mathbf{1}$.

Proof. Clearly 1 is in the kernel of all $\iota_{\zeta}$. Suppose $x \in \Lambda^{*} V_{n}^{\prime}$, and the highest degree summand of $x$ has degree $i>0$. Let $\left\{\zeta_{1}, \ldots, \zeta_{n}\right\}$ be a set of generators of $V_{n}$, and let $\left\{\zeta_{1}^{\prime}, \ldots, \zeta_{n}^{\prime}\right\}$ be a basis of $V_{n}^{\prime}$ such that $\zeta_{i}^{\prime}\left(\zeta_{j}\right)=\delta_{i j}$. Without loss of generality, we can assume $x$ contains a term $k \zeta_{1}^{\prime} \wedge \zeta_{2}^{\prime} \wedge \cdots \wedge \zeta_{i}^{\prime}$, where $k \in \mathbb{Z} \backslash\{0\}$, then

$$
\iota_{\zeta_{1}}(x)=k \zeta_{2}^{\prime} \wedge \cdots \wedge \zeta_{i}^{\prime}+\cdots
$$

is nonzero. This finishes our proof. 
Proposition 4.2. Suppose $K \subset Y_{n}$ is a null-homologous knot with simple Floer homology, and $Y_{n}-K$ is irreducible, then the genus of $K$ is $g=\frac{n}{2}$ and $K$ is fibered.

Proof. Let $F$ be a minimal genus Seifert surface of genus $g$. By definition

$$
A_{\zeta}: \widehat{C F}\left(Y_{n}\right) \rightarrow \widehat{C F}\left(Y_{n}\right)
$$

is a filtered map, so $\widehat{C F K}\left(Y_{n}, K,[F],-g\right)$ is a subcomplex of $\widehat{C F}\left(Y_{n}\right)$. Moreover, since $K$ has simple Floer homology group, the rank of $\widehat{H F K}\left(Y_{n}, K\right)$ is the same as the rank of $\widehat{H F}\left(Y_{n}\right)$, so $\widehat{H F K}\left(Y_{n}, K,[F],-g\right)$ is a submodule of the $\Lambda^{*} V_{n}$-module $\widehat{H F}\left(Y_{n}\right)$. Similarly, $\widehat{H F K}\left(Y_{n}, K,[F], g\right)$ is a quotient module of $\widehat{H F}\left(Y_{n}\right)$.

Let $\operatorname{Ker} A$ be the subgroup of $\widehat{H F K}\left(Y_{n}, K,[F],-g\right)$ which is the intersection of $\operatorname{ker} A_{\zeta}$ for all $\zeta \in V_{n}$, then Lemma 4.1 shows that the rank of $\operatorname{Ker} A$ is at most 1 . It follows from Corollary 1.2 that $K$ is a fibered knot with fiber $F$, and the bottommost summand $\overline{H F K}\left(Y_{n}, K,[F],-g\right)$ is generated by 1.

Any monomial $\zeta_{i_{1}}^{\prime} \wedge \cdots \wedge \zeta_{i_{k}}^{\prime} \in \widehat{H F}\left(Y_{n}\right)$ can be obtained by applying a series of $A_{\zeta}$ 's to $\Delta=\zeta_{1}^{\prime} \wedge \cdots \wedge \zeta_{n}^{\prime}$. Since $A_{\zeta}$ is a filtered map, we see that $\Delta$ has the highest filtration, hence $\widehat{H F K}\left(Y_{n}, K,[F], g\right)$ is generated by the image of $\Delta$ under the quotient map $\widehat{H F}\left(Y_{n}\right) \rightarrow \widehat{H F K}\left(Y_{n}, K,[F], g\right)$.

By [15, Proposition 3.10], the difference between the Maslov grading of $\Delta$ and the Maslov grading of $\mathbf{1}$ is $2 g$. On the other hand, since

$$
\mathbf{1}=A_{\zeta_{1}} \circ \cdots \circ A_{\zeta_{n}}(\Delta)
$$

the difference between the Maslov grading of $\Delta$ and the Maslov grading of $\mathbf{1}$ is $n$. So $n=2 g$.

Proof of Theorem 1.3. If $Y_{n}-K$ is reducible, then it has a $S^{1} \times S^{2}$ connected summand. We can remove this summand and regard $K$ as a knot in $Y_{n-1}$, which still has simple Floer homology group. Hence we may assume that $Y_{n}-K$ is irreducible. By Proposition $4.2, n=2 g$ where $g$ is the genus of $K$, and $K$ is fibered. Let $F$ be the Seifert surface of $K$ which is a fiber of the fibration. Pick a base point on $\partial F$. Let $\varphi: F \rightarrow F$ be the monodromy of the fibration such that $\left.\varphi\right|_{\partial F}$ is the identity. Let $\varphi_{*}: \pi_{1}(F) \rightarrow \pi_{1}(F)$ be the induced map on $\pi_{1}(F)$. Let $t$ represent a meridian of $K$, then

$$
\pi_{1}\left(Y_{2 g}-K\right)=\left\langle\pi_{1}(F), t \mid t \varphi_{*}(a) t^{-1} a^{-1}=1, \forall a \in \pi_{1}(F)\right\rangle
$$


After filling $Y_{2 g}-K$ along the meridian, $t$ is killed, so we get

$$
\pi_{1}\left(Y_{2 g}\right)=\left\langle\pi_{1}(F) \mid \varphi_{*}(a) a^{-1}=1, \forall a \in \pi_{1}(F)\right\rangle,
$$

which is a quotient group of $\pi_{1}(F)$. We know that $\pi_{1}(F)$ is a free group of rank $2 g$. On the other hand, $Y_{2 g}$ is the connected sum of $2 g$ copies of $S^{1} \times S^{2}$, so its $\pi_{1}$ is also a free group of rank $2 g$. Since free groups of finite ranks are Hopfian [11], the relations in (5) are all trivial, hence $\varphi_{*}=\mathrm{id}$.

Now it is a standard fact that $\varphi$ is isotopic to the identity map on $F$ through maps which fix $\partial F$ pointwise. In fact, we define a map

$$
\Phi:(F \times\{0,1\}) \cup(\partial F \times[0,1]) \rightarrow F
$$

by letting

$$
\Phi(x, 0)=\varphi(x), \Phi(x, 1)=x, \quad \forall x \in F
$$

and

$$
\Phi(x, t)=x, \quad \forall x \in \partial F .
$$

Since $\varphi_{*}=$ id and $F$ is a $K(\pi, 1)$, we can extend $\Phi$ to a map from $F \times I$ to $F$. This means that $\varphi$ is homotopic hence isotopic to the identity map relative to $\partial F$.

Since the monodromy $\varphi$ is isotopic to the identity, the complement of $K$ is homeomorphic to $F \times S^{1}$, which is homeomorphic to the complement of $B_{g}$. Since there is only one Dehn filling on $F \times S^{1}$ that yields $Y_{2 g}$, (all other Dehn fillings yield Seifert fibered spaces,) $K=B_{g}$.

\section{Links in $S^{3}$}

In this section, we will study links in $S^{3}$ that have simple Floer homology groups. Ozsváth and Szabó [17] defined a multi-graded $\mathbb{Z} / 2 \mathbb{Z}$-coefficient homology theory for links, called link Floer homology, denoted $\widehat{H F L}(\cdot)$. Although link Floer homology generally contains more information than the knot Floer homology of a link, the rank of $\widehat{H F L}(L)$ is equal to the rank of $\widehat{H F K}(L)$ [17, Theorem 1.1]. So Proposition 1.4 can also be stated in terms of link Floer homology. In fact, we will mainly work with link Floer homology in our proof.

Proof of Proposition 1.4. Without loss of generality, we will work with $\mathbb{F}=$ $\mathbb{Z} / 2 \mathbb{Z}$ coefficients. We will induct on the number of components of $L$. 
When $n=|L|=1$, the result is a consequence of Ozsváth and Szabó [14]. Assume that our result is already proved for $(n-1)$-component links and let $L$ be an $n$-component link such that

$$
\operatorname{rank} \widehat{H F K}(L)=\operatorname{rank} \widehat{H F L}(L)=2^{n-1} .
$$

If $L$ has a trivial component which bounds a disc in the complement of $L$, then we can remove this component and apply the induction hypothesis to conclude that $L$ is the unlink. From now on, we assume $L$ has no trivial component.

Let $K_{1}$ be a component of $L$. Let $M$ be the rank two graded vector space with one generator in grading 0 and another in grading -1 . By Ozsváth and Szabó [17, Proposition 7.1], there is a differential $D^{1}$ on $\overline{H F L}(L)$, such that the homology of $\left.\widehat{(H F L}(L), D^{1}\right)$ is $\widehat{H F L}\left(L-K_{1}\right) \otimes M$. Here the two Floer homology groups have Alexander gradings in $\operatorname{Spin}^{c}\left(L-K_{1}\right)$, and the isomorphism is up to some overall translation of the gradings. So the rank of $\widehat{H F L}\left(L-K_{1}\right)$ is less than or equal to $2^{n-2}$.

Since $L-K_{1}$ is an $(n-1)$-component link, the rank of its link (knot) Floer homology is greater than or equal to $2^{n-2}$, so the rank should be exactly $2^{n-2}$. Hence the differential $D^{1}=0$ and

$$
\widehat{H F L}(L) \cong \widehat{H F L}\left(L-K_{1}\right) \otimes M
$$

up to an overall translation of the gradings, where the Alexander gradings are in $\operatorname{Spin}^{c}\left(L-K_{1}\right)$. By the induction hypothesis, $L-K_{1}$ is the $(n-1)$ component unlink, hence its $\widehat{H F L}$ is supported in exactly one Alexander grading. It follows that $\widehat{H F L}(L)$ is supported in exactly one element in $\operatorname{Spin}^{c}\left(L-K_{1}\right)$, thus $\widehat{H F L}(L)$ is supported in one line in $\operatorname{Spin}^{c}(L)$. Now Ozsváth-Szabó [18, Theorem 1.1] implies that there exists a nonzero element $h \in H^{1}\left(S^{3}-L ; \mathbb{Z}\right)$, such that

$$
x(\mathrm{PD}[h])+\sum_{i=1}^{n}\left|\left\langle h, \mu_{i}\right\rangle\right|=0,
$$

where $\mu_{i}$ is the meridian of the $i$ th component of $L$. Thus $\left|\left\langle h, \mu_{i}\right\rangle\right|=0$ for each $i$, which is impossible since $h \neq 0$, a contradiction.

\section{Acknowledgments}

This work was carried out when the author participated the "Homology Theories of Knots and Links" program at MSRI and when the author visited 
Princeton University. The author wishes to thank MSRI and David Gabai for their hospitality. The author was partially supported by an AIM Five-Year Fellowship and NSF grant number DMS-1021956.

\section{References}

[1] Open problems in geometric topology, in 'Low-dimensional and symplectic topology, Proc. 2009' (M. Usher, ed.), Georgia Int. Topology Conf. held at the University of Georgia, Athens, GA, May 18-29, 2009, 'Proc. Symp. Pure Mathematics', 82, American Mathematical Society, Providence, RI, 2011, 215-228.

[2] D. Gabai, Foliations and the topology of 3-manifolds, J. Differential Geom. 18(3) (1983), 445-503.

[3] - Foliations and the topology of 3-manifolds II, J. Differential Geom. 26(3) (1987), 461-478.

[4] P. Ghiggini, Knot Floer homology detects genus-one fibred knots, Amer. J. Math. 130(5) (2008), 1151-1169.

[5] M. Hedden, On Floer homology and the Berge conjecture on knots admitting lens space surgeries, Trans. Amer. Math. Soc. 363(2) (2011), 949-968.

[6] M. Hedden and Y. Ni, Khovanov module and the detection of unlinks, Geom. Topol. 17(5) (2013), 3027-3076.

[7] K. Honda, W. Kazez and G. Matić, Convex decomposition theory, Int. Math. Res. Not. (2002), (2), 55-88.

[8] — The contact invariant in sutured Floer homology, Invent. Math. 176(3) (2009), 637-676.

[9] A. Juhász, Holomorphic discs and sutured manifolds, Algebr. Geom. Topol. 6 (2006), 1429-1457.

[10] - Floer homology and surface decompositions, Geom. Topol. 12(1) (2008), 299-350.

[11] W. Magnus, A. Karrass and D. Solitar, Combinatorial group theory: presentations of groups in terms of generators and relations, Dover Publications, Inc., New York, 2nd revised edn., 1976.

[12] Y. Ni, A note on knot Floer homology of links, Geom. Topol. 10 (2006) 695-713. 
[13] - Knot Floer homology detects fibred knots, Invent. Math. 170(3) (2007), 577-608;

Erratum, Invent. Math. 177(1) (2009), 235-238.

[14] P. Ozsváth and Z. Szabó, Holomorphic disks and genus bounds, Geom. Topol. 8 (2004), 311-334.

[15] - Holomorphic disks and knot invariants, Adv. Math. 186(1) (2004), 58-116.

[16] - Holomorphic disks and topological invariants for closed threemanifolds, Ann. Math. (2) 159(3) (2004), 1027-1158.

[17] - Holomorphic disks, link invariants and the multivariable Alexander polynomial, Algebr. Geom. Topol. 8(2) (2008), 615-692.

[18] - Link Floer homology and the Thurston norm, J. Amer. Math. Soc. 21(3) (2008), 671-709.

[19] Knot Floer homology and rational surgeries, Algebr. Geom. Topol. 11(1) (2011), 1-68.

[20] J. Rasmussen, Floer homology and knot complements, Ph.D. thesis, Harvard University (2003). Available at, arXiv:math.GT/0306378.

[21] - Lens space surgeries and L-space homology spheres (2007). Available at arXiv:math.GT/0710.2531.

[22] S. Sarkar and J. Wang, An algorithm for computing some Heegaard Floer homologies, Ann. Math. (2) 171(2) (2010), 1213-1236.

Department of Mathematics

Caltech

MC 253-37, 1200 E CALIFornia Blvd

PASADENA, CA 91125

USA

E-mail address: yini@caltech.edu

RECEIVED MAY 4, 2013 
